\title{
АБСОЛЮТНЫЕ РАЗНОСТИ ФАЗ В ИНТЕГРАЛЬНОЙ ФОТОУПРУГОСТИ
}

\author{
(Представил Х. Абен)
}

\section{1. Введение}

В интегральной фотоупругости $\left[{ }^{1}\right]$ трехмерное напряженное состояние модели определяется на основе интегральных оптических величин.

Обычно используют характеристические величины или параметры изоклины и относительные разности хода. В некоторых случаях при определении напряженного состояния можно использовать априорную информацию о связи между компонентами тензора (уравнения совместности, уравнения равновесия) [ $\left.{ }^{1}\right]$. В качестве дополнительной информации можно использовать также отклонения световых лучей $\left[{ }^{2}\right]$. Однако информащия, получаемая измерением отклонения световых лучей, несколько беднее, чем информация, получаемая измерением абсолютных разностей хода в результате операции дифференцирования.

В настоящей работе изучаются возможности, которые дает измерение абсолютных разностей фаз в интегральной фотоупругости.

\section{2. Абсолютные разности фаз}

Преобразования световых векторов в непоглощающей фотоупругой среде описываются унитарной унимодулярной матрицей $\left[{ }^{1}\right]$, которую можно записать в виде:

$$
U=\left(\begin{array}{rl}
e^{i \xi} \cos \theta & e^{i \xi} \sin \theta \\
-e^{-i \xi} \sin \theta & e^{-i \xi} \cos \theta
\end{array}\right)
$$

где $\xi$, $\zeta$ и $\theta$ параметры среды для рассматриваемого луча. Используя матрицу (1), преобразование световых векторов в фотоупругой среде можно записать в виде:

$$
\vec{E}_{*}=U \vec{E}_{0}
$$

где $\vec{E}_{0}$ и $\vec{E}_{*}$ - входящие в фотоупругую среду и выходящие из нее световые векторы соответственно в единой системе координат. Матрица $U$ не позволяет учитывать абсолютных разностей фаз. Умножим каждый элемент матрицы (1) на $e^{i \delta}$, где $\delta$ какой-нибудь новый неза-

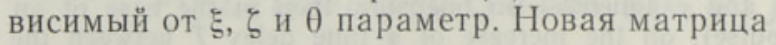

$$
U^{\prime}=e^{i \delta} U \text {, }
$$

как это легко доказать прямой проверкой, также является унитарной.

Как показано в $\left[{ }^{1}\right]$, если не учитывать абсолютных разностей фаз, матрица преобразования (1) зависит максимально от трех параметров. Если мы используем матрицу вида (3), которая зависит от четырех параметров, то добавлением члена $e^{i \delta}$ мы вводим абсолютные фазы. 
Все выводы, сделанные в оптической теории неоднородной фотоупругой среды, остаются такими же, как в [ $\left.{ }^{1}\right]$.

Если мы задаем $\vec{E}_{0}$ и $\vec{E}_{*}$ в характеристических направлениях [ $\left.{ }^{1}\right]$, то матрица $U^{\prime}$ приобретает диагональный вид:

$$
U^{\prime}=\left(\begin{array}{cc}
e^{i(\delta+\gamma)} & 0 \\
0 & e^{i(\delta-\gamma)}
\end{array}\right),
$$

где величину $2 \gamma$ называют характеристической разностью фаз [ $\left.{ }^{1}\right]$. Величины $\delta_{1}$ и $\delta_{2}$, определяемые формулами:

$$
\begin{aligned}
& \delta_{1}=\delta+\gamma, \\
& \delta_{2}=\delta-\gamma,
\end{aligned}
$$

будем называть абсолютными характеристическими разностями фаз. Они, как и $2 \gamma$, относятся к компонентам светового вектора, которые параллельны характеристическим направлениям. В случае двумерных моделей $\delta_{1}$ и $\delta_{2}$ совпадают с абсолютными разностями фаз, приведенными в $\left[{ }^{3}\right]$.

Из (5) и (6) следует

$$
\delta_{1}+\delta_{2}=2 \delta .
$$

Величину $\delta$ называем абсолютной разностью фаз неполяризованного света. Действительно, преобразование световых векторов членом $e^{i \delta}$ не зависит от направления падающих световых векторов. Таким образом, параметр $\delta$ характеризует среду независимо от того, является свет поляризованным или нет. Это обстоятельство особо важно при экспериментальном определении параметра $\delta$.

\section{3. Экспериментальное определение абсолютной разности фаз}

Голографические и интерферометрические методы исследования прозрачных фазовых объектов можно разделить на поляризационные методы (например, голографическая фотоупругость) и на методы, где результаты измерения не зависят от поляризации (например, изучение потоков газа). В голографической или интерферометрической фотоупругости в основном изучались плоские задачи $\left[{ }^{3-6}\right]$. Главные проблемы в экспериментальных исследованиях связаны с интерпретацией полос. Поляризационные методы дают одновременно два семейства полос $\left[{ }^{7-9}\right]$ : изохромы, представляющие собой линии постоянной величины разности главных напряжений, и изопахики, являющиеся линиями постоянного значения суммы главных направлений. Однако существуют методы $\left[{ }^{10-13}\right]$, которые дают картины изохром и изопахик раздельно. Чтобы получить картину изопахик, модель просвечивают дважды (в прямом и обратном направлениях), причем между просвечиваниями плоскость поляризации света поворачивают на 90\%. Это реализовано в $\left[{ }^{10}\right]$ при помощи магнитного поля и в $\left[{ }^{11-13}\right]$ различными другими оптическими ротаторами.

Рассмотрим применение такого метода $\left[{ }^{11-13}\right]$ в случае трехмерных моделей. Используем матрицу $U^{\prime}$ для описания преобразования световых вектиров при «прямом» просвечивании. Поворот световых векторов на $90^{\circ}$ опишем матрицей вида:

$$
R=\left(\begin{array}{rr}
\cos 90^{\circ} & -\sin 90^{\circ} \\
\sin 90^{\circ} & \cos 90^{\circ}
\end{array}\right)=\left(\begin{array}{rr}
0 & -1 \\
1 & 0
\end{array}\right)
$$

Прохождение света через среду в обратном направлении описывает транспонированная матрица $\widetilde{U}^{\prime}\left[{ }^{1}\right]$. Таким образом, преобразование световых векторов в рассматриваемой системе описывается матрицей

$$
K=\widetilde{U}^{\prime} R U^{\prime}
$$


Подставляя $U^{\prime}$ из формулы (3) в формулу (9), получим

$$
K=e^{i 2 \delta}\left(\begin{array}{rr}
0 & -1 \\
1 & 0
\end{array}\right) \text {. }
$$

Пусть световые векторы

$$
\vec{E}_{*}^{\prime}=K \vec{E}_{0}
$$

интерферируют с векторами $\vec{E}_{0}$. После интерференции преобразование световых векторов можно описать формулой

$$
\vec{E}_{*}^{\prime \prime}=M \vec{E}_{0},
$$

где матрица преобразования $M$ приобретает вид:

$$
M=\left(\begin{array}{cc}
1 & -e^{i 2 \delta} \\
e^{i 2 \delta} & 1
\end{array}\right) .
$$

Используя (12) и (13), можно записать интенсивность света, выходящего из системы (при циркулярно поляризованной падающей волне), по формуле

$$
I=I_{0} \cos ^{2}\left(\delta+45^{\circ}\right) .
$$

Из (14) видно, что по интерференционной картине в случае трехмерного напряженного состояния можно определить величину $\delta$. При двумерном напряженном состоянии $\delta$ совпадает с абсолютными разностями фаз, приведенными в [10-13].

Вышеописанные методы для определения $\delta$ являются сложными, поскольку они требуют специальной экспериментальной установки с ротатором, который поворачивает плоскость поляризации света на $90^{\circ}$. Экспериментальные сложности можно преодолеть, если модель изготовлена из оптически малочувствительного материала. В таком случае изохромы не появляются или мало влияют на картину изопахик $\left[{ }^{10}\right]$. Другим способом можно определить изопахики отдельно, используя неполяризованный свет.

При изучении двухмерных моделей на основе картины изохром можно получить распределение разности хода. Поэтому в таком случае целесообразно получить методом $\left[{ }^{10-13}\right]$ отдельно картины изохром и изопахик. Однако при трехмерных моделях в общем случае надо знать три характеристические величины, и информации, получаемой от одной картины изохром, не хватает для установления распределения характеристической разности хода, не говоря о характеристических углах. Поэтому здесь целесообразно измерить все характеристические величины отдельно и для определения $\delta$ использовать «неполяризационные» методы голографии или интерферометрии.

В $\left.{ }^{4}\right]$ приведен анализ оптических явлений в трехмерных моделях, используя приближение геометрической оптики. Там получены четыре различных компонента оптических путей $\varphi_{1}, \varphi_{2}, \psi_{1}, \psi_{2}$, где $\varphi_{1}, \varphi_{2}$ получены в нулевом приближении и $\psi_{1}, \psi_{2}$ в первом приближении. Разности фаз выражаются в виде:

$$
\begin{aligned}
& \delta_{1}=\left(\varphi_{1}+\psi_{1}\right) \frac{2 \pi}{\lambda}, \\
& \delta_{2}=\left(\varphi_{2}+\psi_{2}\right) \frac{2 \pi}{\lambda} .
\end{aligned}
$$

Однако формулы (15) и (16) получены для сильно анизотропных сред. В слабоанизотропных средах метод геометрической оптики 
можно упростить. В $\left[{ }^{15}\right]$ считая, что в нулевом приближении среда является изотропной, получены формулы

$$
\begin{aligned}
& \delta_{1}=\left(\varphi+\psi_{1}\right) \frac{2 \pi}{\lambda}, \\
& \delta_{2}=\left(\varphi+\psi_{2}\right) \frac{2 \pi}{\lambda},
\end{aligned}
$$

где $\varphi$ - эйконал изотропной среды. Формулы (17) и (18) в характеристических направлениях приобретают вид (5) и (6).

Как показывает формула (14), на основе интерференционной картины можно получить только одну физическую величину $\delta$. В фотоупругости нет никакого основания разбивать эту величину на два компонента как это сделано в $\left[{ }^{14}\right]$.

\section{4. Соотношения между $\delta$ и напряжениями}

Трехмерную фотоупругую среду можно представить как стопу поставленных друг за другом двупреломляющих пластинок. Матрицу $U_{j}$ элемента $j$ стопы, описывающей преобразование светового эллипса в двупреломляющем слое, можно представить в виде:

$$
U_{j}=e^{i \delta_{j}} U_{0 j},
$$

где $U_{0 j}$ - матрица слоя преобразования световых векторов, не учитывающая абсолютных фаз, $\delta_{j}-$ абсолютная фаза слоя. Матрица всей стопы определяется соотношением

$$
U=U_{0 n} U_{0 n-1} \ldots U_{0 j} \ldots U_{0 n} U_{01} e^{i\left(\delta_{1}+\delta_{2}+\cdots \delta_{j}+\cdots \delta_{n-1}+\delta_{n}\right)} .
$$

Выразим главные показатели преломления слоя через

$$
\begin{aligned}
& n_{1 j}=n_{0}+\Delta n_{1 j}, \\
& n_{2 j}=n_{0}+\Delta n_{2 j},
\end{aligned}
$$

где $n_{0}$ - показатель преломления ненапряженной среды, $\Delta n_{1 j}$ и $\Delta n_{2 j}$ - изменения показателя преломления вследствие напряжений. Величина $\delta_{j}$ выражается через $n_{1 j}$ и $n_{2 j}$ следующим образом (двумерный случай):

$$
\delta_{j}=\frac{2 \pi}{\lambda}\left(2 n_{0}+\Delta n_{1 j}+\Delta n_{2 j}\right) \Delta y_{j}
$$

где $\Delta y_{j}$ - толщина слоя, $\lambda$ - длина волны.

Используя (20) и (22), получим

$$
\delta=\sum_{j=1}^{n} \delta_{j}=\sum_{j=1}^{n} \frac{2 \pi}{\lambda}\left(2 n_{0}+\Delta n_{1 j}+\Delta n_{2 j}\right) \Delta y_{j} .
$$

Если толщина слоя $\Delta y_{j}$ приближается к нулю, то получим

$$
\delta=\frac{2 \pi}{2} \int_{y_{0}}^{*}\left(2 n_{0}+\Delta n_{1}+\Delta n_{2}\right) d y,
$$

где $y_{0}$ и $y_{*}$ - соответственно координаты точек вхождения света в модель и выхода света из модели.

В двухэкспозиционной голографии определяют $\delta$ относительно начального значения $\delta_{0}$. Это позволяет определить абсолютную разность фаз 


$$
\delta-\delta_{0}=\frac{2 \pi}{\lambda} \cdot\left[\int_{y_{0}}^{y_{*}}\left(2 n_{0}+\Delta n_{1}+\Delta n_{2}\right) d y-\int_{y_{0}}^{y_{*}^{\prime}} 2 n_{i} d y-\int_{y_{0}^{\prime}}^{y_{*}^{\prime}} 2 n_{0} d y-\int_{y_{*}^{\prime}}^{y_{*}} 2 n_{i} d y\right]
$$

где $n_{i}$ - показатель преломления окружающей среды и $y_{0}{ }^{\prime}, y^{*^{\prime}}$ координаты точек входа света в модель и выхода света из модели после загружения. Величина

$$
\Delta l=y_{0}^{\prime}-y_{0}+y_{*}-y_{*}^{\prime}
$$

дает изменение толщины модели. Формулу (25) можно выписать в виде:

$$
\delta-\delta_{0}=\frac{2 \pi}{\lambda}\left(n_{0}-n_{i}\right) 2 \Delta l+\frac{2 \pi}{\lambda} \int_{y_{0}}^{y^{*}}\left(\Delta n_{1}+\Delta n_{2}\right) d y .
$$

Имея в виду, что оптическая анизотропия является малой, можем рассматривать показатель преломления как тензор. На распространение света влияют только два компонента тензора показателя преломления, которые находятся в плоскости, перпендикулярной лучу. Под интегралом в формуле (27) стоит первый инвариант этого тензора

$$
\Delta n_{1}+\Delta n_{2}=\Delta n_{x x}+\Delta n_{z z},
$$

где $\Delta n_{x x}$ и $\Delta n_{z z}$ - компоненты тензора показателя преломления, параллельные осям $x$ и $z$.

Запишем закон Максвелла в виде:

$$
\begin{aligned}
& \Delta n_{x x}=c_{1} \sigma_{x x}+c_{2}\left(\sigma_{y y}+\sigma_{z z}\right), \\
& \Delta n_{z z}=c_{1} \sigma_{z z}+c_{2}\left(\sigma_{x x}+\sigma_{y y}\right),
\end{aligned}
$$

где $c_{1}$ и $c_{2}-$ упруго-оптические постоянные.

Запишем также закон Гука

$$
\Delta l=\frac{1}{E} \int_{y_{0}}^{y_{*}}\left[\sigma_{y y}-\mu\left(\sigma_{x x}+\jmath_{z z}\right)\right] d y,
$$

где $E$ - модуль упругости материала, $\mu$ - коэффициент Пуассона.

Используя (28)-(30), можем формулу (27) привести к виду:

$$
\delta-\delta_{0}=A \int_{y_{0}}^{y_{*}} \sigma_{y y} d y+B \int_{y_{0}}^{y_{*}}\left(\sigma_{x x}+\sigma_{z z}\right) d y
$$

где

$$
\begin{aligned}
& A=\left[2\left(n_{0}-n_{i}\right) \frac{1}{E}+2 c_{2}\right] \frac{2 \pi}{\lambda}, \\
& B=\left[c_{1}+c_{2}-2\left(n_{0}-n_{i}\right) \frac{\mu}{E}\right] \frac{2 \pi}{\lambda} .
\end{aligned}
$$

При двумерном состоянии напряжений $\sigma_{y y}$ равняется нулю и формула (31) совпадает с формулой, приведенной в [ $\left.{ }^{3}\right]$.

Если иммерсия точная, $n_{i}=n_{0}$, и, если $c_{1}=c_{2}$, то можно определить первый инвариант тензора напряжений $\left[{ }^{16}\right]$.

Из формулы (31) видим, что $\delta$ выражается через компоненты напряжений простым интегральным соотношением. Отметим, что в интегральной фотоупругости параметры среды $\xi$, $\zeta$ и $\theta$ выражаются через напряжения сложным образом. 


\section{5. Заключение}

Показано, что дополнительное измерение в интегральной фотоупругости абсолютных разностей фаз $\delta$ дает одно дополнительное соотношение (31) для определения напряжений в общем трехмерном случае. В интегральной фотоупругости без предположения, что вращение квазиглавных направлений малое, система интегральных уравнений, связывающая напряжения с характеристическими величинами, решается только численно. Формула, связывающая напряжения с абсолютными разностями фаз, таких ограничений не имеет.

\section{Л ИТ ЕРАТУРА}

1. Абен Х. К. Интегральная фотоупругость. Таллин, Валгус, 1978.

2. Абен Х. К., Келл К.-Ю. Э. // Прнкл. мех., 1985, 21, № 9, 11-15.

3. Dhir, S. K., Peterson, H. A. // Exp. Mech., 1971, 11, № 12, 560-564.

4. Ajovalasit, A., Bardi, A. // Exp. Mech., 1976, 16, № 7, 273-275.

5. Sciammarella, C. A., Quintanilla, G. // Exp. Mech., 1972, 12, № 2, 57-66.

6. Chih-Kung Lee, Ching-Piao Hu // Appl. Opt., 1986, 25, № 12, 1912-1916.

7. Sanford, R. Y., Durelli, A. J. // Exp. Mech., 1971, 11, № 4, 161-166.

8. Hovanesian, J. der // Exp. Mech., 1974, 14, № 6, 233-236.

9. Ebbeni, J., Coenen, J., Hermanne, A. // J. Strain Anal., 1977, 11, № 1.

10. Chau Henry, H. M. // The Rev. of Scient. Instr., 1968, 39, № 12, 1789-1792.

11. O'Regan, R., Dudderar, T. D. // Exp. Mech., 1971, 11, № 6, 241-247.

12. Assa, A., Betser, A. A. // Exp. Mech., 1974, 14, № 12, 502-504.

13. Kjell Gasvik // Exp. Mech., 1976, 16, № 4, 146-150.

14. Зимин В. Д., Шахурдин В. И. // Прикл. мех., 1978, 14, № 5, 25-29.

15. Кравцов Ю. И., Орлов Ю. И. Геометрическая оптика неоднородных сред. М., Наука, 1980.

16. Vest, C. M., Ural, E. A. The Role of Interferometry and Tomography in Stress Analysis of Transparent Media: Proc. SESA Spring Meeting. Dearborn, 1981, $242-245$.

Институт кибернетики

Академии наук Эстонской ССР
Поступила в редакцию $8 /$ II 1988

\section{K.-J. KELL}

\section{ABSOLUUTSED FAASIVAHED INTEGRAALSES FOTOELASTSUSES}

On vaadeldud vōimalusi, mida annab absoluutsete faasivahede mõõtmine integraalses fotoelastsuses, ning näidatud, et isopahhide pildi alusel on võimalik mõôta absoluutseid faase, kui on tegemist mudeliga, milles on kolmemõōtmeline pingeolukord. On toodud valem, mis seob absoluutseid faasivahesid pinge komponentidega.

\section{K.-J. KELL}

\section{ABSOLUTE PHASE RETARDATIONS IN INTEGRATED PHOTOELASTICITY}

The measurement of absolute retardations in the field of integrated photoelasticity may give us some new valuable information about the body under investigation. In this paper the measurement and utilization of absolute retardation is considered.

It is shown that in the case of three-dimensional state of stress the «isopachic pattern» gives us the integrals of absolute retardation. An expression that connects the components of stress tensor with absolute retardation has been derived. 